\title{
The Modified Gaussian Function based RED (MGF-RED) Algorithm for Congestion Avoidance in Mobile Ad Hoc Networks
}

\author{
Makul Mahajan \\ Student, Dept. of CSE \\ Amritsar College of Engineering \& Technology \\ PTU, Jalandhar, Punjab
}

\author{
Tanu Preet Singh, Ph.D \\ Professor and HOD, Dept. of ECE \\ Amritsar College of Engineering and Technology, \\ PTU, Jalandhar, Punjab
}

\begin{abstract}
In this paper the idea is to rationale a new active queue management technique (MGF-RED) based on Random Early Detection (RED), a well known active queue management technique for congestion avoidance. The conventional RED uses the concept of minimum threshold, maximum threshold and average queue length. If the average queue length increases by minimum threshold then the algorithm start dropping the packets with some probability using linear function of average queue size. If the average queue size is greater than the maximum threshold then packets are dropped with 1 probability. The proposed algorithm will use Modified Gaussian function for calculating the likelihood of packet drop as the average queue length increases the minimum threshold. Simulation has been performed using NS-2.34. The simulation results show that the proposed algorithm will present better network performance in throughput, packet delivery fraction and number of dropped packets.
\end{abstract}

\section{General Terms}

MANET, RED, Gaussian Function.

\section{INTRODUCTION}

A Mobile Ad Hoc network consists of various data transmission mobile nodes that can forward the packet from one node to another node. There is no permanent infrastructure in mobile ad hoc network. Communication between the nodes takes place by the mean of wireless links [1]. Due to this property of mobile ad hoc network, the network can be setup at any time and at anywhere. As the nodes in this type of network are mobile therefore the topology is highly dynamic. The topology of the mobile ad hoc network depends upon the mobility of nodes. If mobility is high then topology of the network changes very rapidly [2][3].

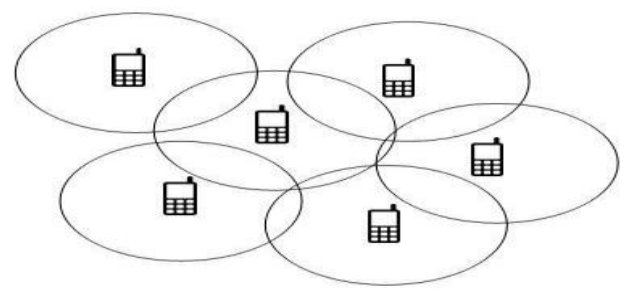

Fig 1: A Mobile Ad Hoc Network with 6 Nodes
Another feature of mobile ad hoc network is that it can perfectly be used in military applications as setting up this type of network is cost effective and takes less time[2] [3]. The one of the challenge in Mobile ad hoc network is providing the QOS.

Each device in MANET has some buffer space provided that can store packets arriving at the queue if the packet cannot be transferred instantaneously [4].

If the queue becomes full, then it leads to congestion hence packet starts dropping. Several techniques are used for avoiding the congestion in the network. Active Queue Management (AQM) [9] is one of the techniques used for managing the buffer space. Another technique that is used for managing the queue space in network is drop tail technique [11], which is passive queue management technique. In drop tail technique the packets are dropped once the queue become full. Drop tail mechanism of queue management suffers from the problem of global synchronization. RED [10] (Random Early Detection) technique is used for managing the queue actively which provides a competence of early congestion by dropping/marking (if ECN is active) the packets as the queue size increases by some threshold. This system reduces the packet loss rate and increases throughput of the network [3][4][5]. Although RED provides a solution for active queue management but the function used in RED algorithm for calculating the probability of dropping the packets when queue size increases by some minimum threshold is linear function of queue average length. Hence the packet loss rate increases as the queue average length increases [4] [5]. So, the work is to propose a function that does not increases linearly with average queue size.

In this paper the main objective is to propose a new Active queue management technique based on modified Gaussian function named MGF-RED. The proposed algorithm will use modified Gaussian function for dropping the packets when the average queue length increases above minimum threshold instead of linear function. The result shows that the proposed algorithm outperforms the traditional RED.

\section{RELATED WORK}

In [4], the authors have discussed the limitation of drop tail queue and proposed a RED with ECN scheme that increases throughput by $10 \%$. In [5], the authors have proposed a new active queue management technique named as adaptive RED based on traditional RED algorithm. In [6], a new improved RED algorithm is proposed that uses packet delay and queue length for calculating the probability of packet drop. In [7], the author has proposed a new fuzzy controller based RED algorithm that is an improvement on traditional RED 
algorithm. In [8], a new AQM algorithm named as PERED is proposed to improve router performance in term of stability of queue size and packet drop.

\section{CONVENTIONAL RED ALGORITHM}

One practice that is used for managing the queue in MANET is Drop Tail Queue [11]. In drop tail technique the algorithm start dropping the packets when the queue becomes full. Drop tail actually work on the principle of FIFO queue in which packets are coming one by one and leave the queue at another end. But if the queue size becomes full then it start dropping the packet hen there is no technique of early congestion notification [4] [8].

Random Early Detection (RED) [10] is well known technique used for active queue management and for congestion avoidance in computer networks [6][7].RED algorithm is sensitive on following parameters:

Average Queue Length (avg_len)

Minimum Threshold (minth)

Maximum Threshold (maxth)

Maximum Drop Probability (maxp)

RED Algorithm first calculates the average queue length and then it compares the average queue length with the minimum and maximum threshold. [4] [8]

If the average queue length (avg_len) is less the minimum threshold then no packet is dropped. If average queue length is greater than minimum threshold(minth) then it checks if average queue length is less than maximum threshold(maxth) then packet drop probability is calculated using the below stated formula:

$$
\mathrm{P}=\mathrm{v} \_\mathrm{a} \text { *avg_len+v_b }
$$

Where $v \_a$ and $v \_b$ are constants and are given by:

$\mathrm{v} \_\mathrm{a}=1 /$ maxthresh-minthresh.

$\mathrm{v} \_\mathrm{b}=\min t h r e s h /($ maxthresh-minthresh).

Avg_len is queue average length.

If the average queue length is greater than maximum threshold then packets are always dropped until queue length reaches the value less than maxthresh.

Following is the basic algorithm used in RED.

1. estimate average queue length (avg_len)

2. if avg_len $<$ minth then,

enque the packet i.e. No drop.

else if avg_len> minth and avg_len<maxth than,

Calculate drop probability using linear function of avg_len.

else if avg_len>maxth then,

Drop the packet always.

3. Exit.

One of the main advantage of using RED over other techniques like Drop Tail, it early detects the congestion before becoming burst. Due to this the queue length can be controlled and hence reduces the packet drop ratio. However from the survey of RED it has observed that the RED algorithm has problem such as low throughput and unfairness to connections. Secondly the packet drop Probability function used in RED is a linear function of average queue length i.e. as the average queue size increases the probability of packet drop also increases[6] [8]. So This paper has proposed a new modified Gaussian based RED which distributes the probability symmetrically rather than linearly. The simulation results show that the proposed modified Gaussian RED outperforms the traditional RED.

\section{PROPOSED ALGORITHM (MODIFIED GAUSSIAN RED)}

This section presents a new active queue management technique named modified Gaussian RED.

From the above discussion it is observed that there is a need of work on linear function which is used for calculating the probability of packet dropping when the average queue length exceeds the minimum threshold but less than maximum threshold [5] [8].

After studying the various probability functions it is decided to include a modified Gaussian function for calculating the probability of packet dropping. Hence the proposed packet dropping probability function is given by:

$$
\mathrm{P}=\mathrm{a}^{*} \mathrm{v} \_\mathrm{a}^{-\mathrm{x}}+\mathrm{d}
$$

Where $\mathrm{x}=(\text { avg_len }-\mathrm{b})^{2} / 2 \mathrm{c}^{2}$

avg_len is the average queue size and is given by:

avg len $=\mathrm{q}$ len* $\mathrm{w}$ and $\mathrm{w}$ is the queue weight and is constant value provided at the time of simulation.

Where $\mathrm{P}$ is new packet drop probability

$\mathrm{v} \_\mathrm{a}$ and $\mathrm{v} \_\mathrm{b}$ are constant and are given by

$\mathrm{v} \_\mathrm{a}=1 /$ maxth-minth

v_b $=$ minth/maxth-minth

$a=\cos ^{2}$ (theta) $+\sin ^{2}$ (theta)

$\mathrm{b}=-\sin (2 *$ theta $)+\sin (2 *$ theta $)$

$\mathrm{c}=\sin ^{2}$ (theta) $+\cos ^{2}$ (theta)

theta $=\mathrm{v} \_\mathrm{a}$ *avg_len+v_b.

In the proposed algorithm as the packet came, the algorithm calculates the average queue length first. Then the average queue length is compared with the minimum and maximum threshold and work in the following steps:

Step 1: calculate the average queue length

Step 2: if average queue length $<$ minimum threshold then Enque the packet

Step 3: if average queue length is $>$ minimum thresholds and $<$ Maximum threshold then

Calculate packet drop probability using above equation (Modified Gaussian probability)

Step 4: if average queue length> maximum threshold then Drop the packet.

Step 5: Exit 
The Proposed Flowchart is:

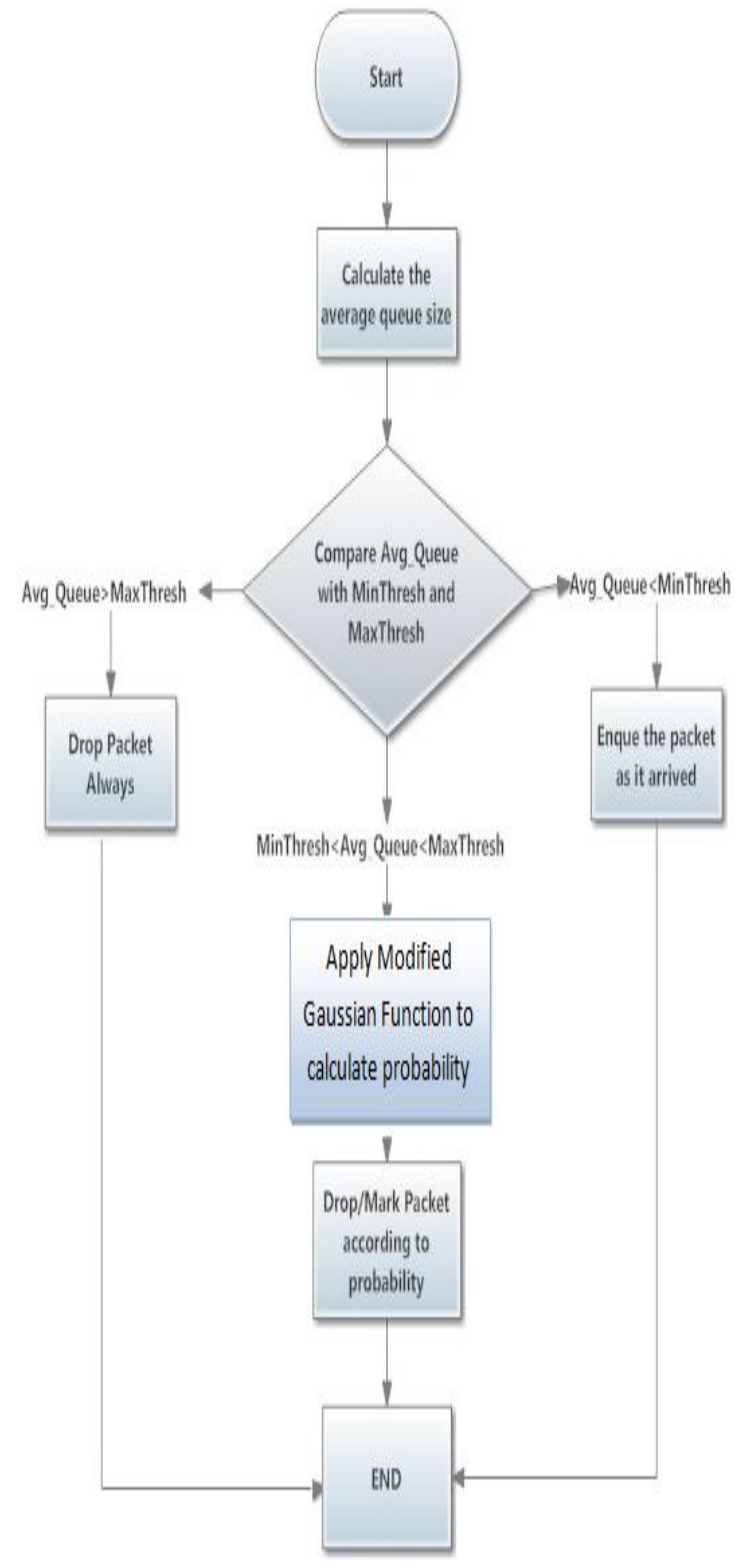

Fig 2: Proposed Flowchart

In the proposed flowchart as shown in figure 2 instead of using the linear function for calculating the probability of packet drop we are using the modified Gaussian function. The modified Gaussian function used for calculating the packet drop probability is a symmetric function. It distributes the probability symmetrically rather than linearly. Hence when the congestion is there the probability of dropping the packet does not increase linearly with the average queue length hence fewer packets are dropped and network performance is improved.
The following figure shows the proposed method:

Case A: An Empty Queue

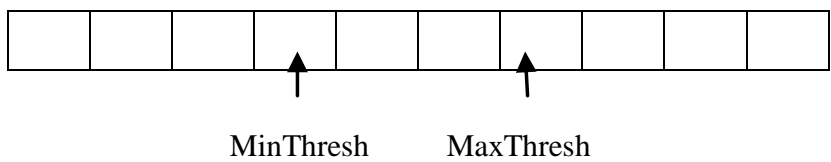

Case B: Avg_que<MinThresh (No Drop)

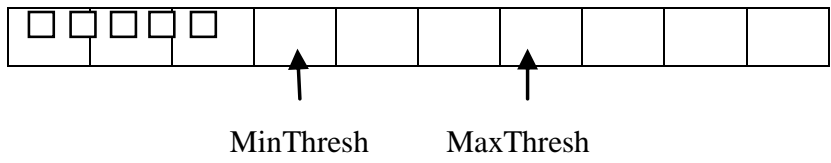

Case C: Avg_que>MinThresh and < MaxThresh (Drop with modified Gaussian probability)

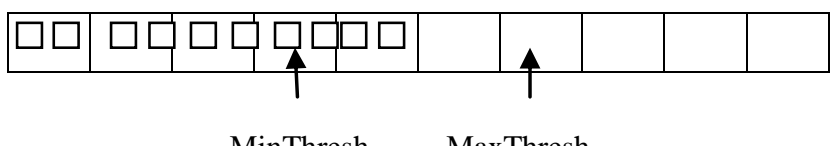

Case D: Avg_que>MaxThresh (Drop the packet until queue become less than MaxThresh)

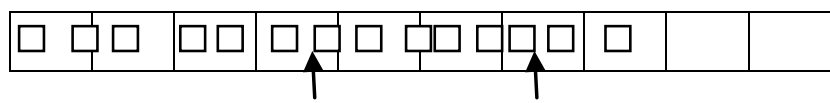

MinThresh MaxThresh

Fig: 3 Proposed Mechanisms

The proposed algorithm as shown in figure 2 for congestion avoidance is the modified version of conventional RED algorithm. In the proposed algorithm when the packet arrives algorithm calculate the average queue length. Then the average queue length is compared with the minimum threshold. If it is less than minimum threshold then packet is processed. If it is more than minimum threshold then compare it with maximum threshold. If average queue length is greater than minth and less than maxth then packet drop probability is calculated using modified Gaussian probability function instead of linear function. If the avg_len is greater than maximum threshold then packet is dropped always.

In short, the objective is to include a new probability function named modified Gaussian probability function for calculating the packet drop probability. The simulation results shows that the proposed algorithm gives increased throughput, increased packet delivery fraction and decreased number. 


\section{SIMULATION SCENARIO}

The simulation has been performed using NS-2.34. In the simulation AODV protocol has been taken as foundation. Initially the procedure calculates the throughput, PDF and End to End delay for RED algorithm then the same procedure is repeated for the modified Gaussian function based RED i.e. the proposed algorithm. The simulation results shows that the proposed algorithm give the better network recital in requisites of throughput, PDF and delay. In the simulation comparison of existing algorithm with the proposed algorithm has been done. The simulation parameters taken are:

Table 1

\begin{tabular}{|l|l|}
\hline Parameter & Value \\
\hline Routing Protocol & AODV \\
\hline Number of Nodes & 30 \\
\hline Pause Time & $6 \mathrm{~s}$ \\
\hline Area & $800 * 800 \mathrm{~m}^{2}$ \\
\hline Speed & $1000 \mathrm{~m} / \mathrm{s}$ \\
\hline Packet Size & $1000 \mathrm{~b}$ \\
\hline Simulation Time & $60 \mathrm{~s}$ \\
\hline Traffic Pattern & CBR \\
\hline Mobility Model & Random Way Point Model \\
\hline
\end{tabular}

The following parameters of RED algorithm need to be set in TCL scripts while executing the conventional and proposed RED algorithm while performing the simulation:

Minimum threshold: 15

Maximum Threshold: 45

Queue weight: 0.002

Maximum drop probability: 0.05

Simulation has been performed by taking the above stated simulation parameters. The trace files have been generated by simulating the scenario and with the help of XGRAPH and AWK files results are formulated.

\section{RESULTS AND DISCUSSION}

The results have been obtained by using the NS-2.34 simulator and results shows that our proposed algorithm performs better than traditional RED algorithm in term of throughput, packet delivery ratio and packet drop rate.

\section{Throughput}

It may be defined as the total amount of data received by destination from the source node divided by time taken to receive the last packet. Figure 4 shows the Simulation Time (X-Axis) versus Throughput (Y-Axis) of MGF-RED and RED. It has been clear from the figure that the proposed algorithm gives the higher throughput than the Traditional RED. The simulation is carried out for $60 \mathrm{sec}$ and it has been observed from the results that the proposed algorithm gives a throughput of 404.65 and RED gives the throughput of 403.95 .

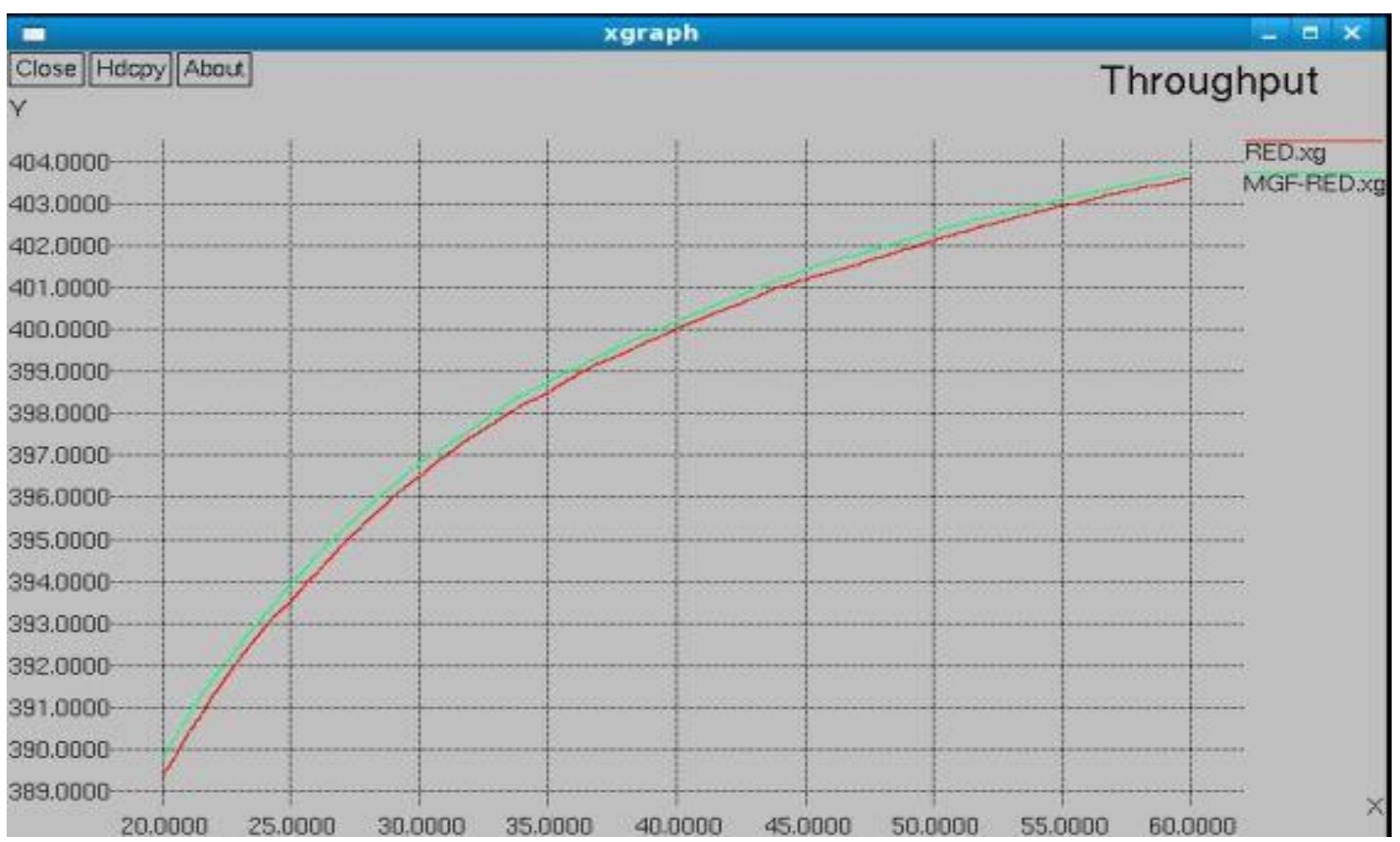

Fig 4: Throughput versus simulation time. 


\section{Packet Delivery Ratio}

It the total number of packets received by destination to total number of packet sent by source. Figure 5 shows the simulation time (X-Axis) versus packet delivery ratio (YAxis) of MGF-RED and RED. The figure 5 shows that the

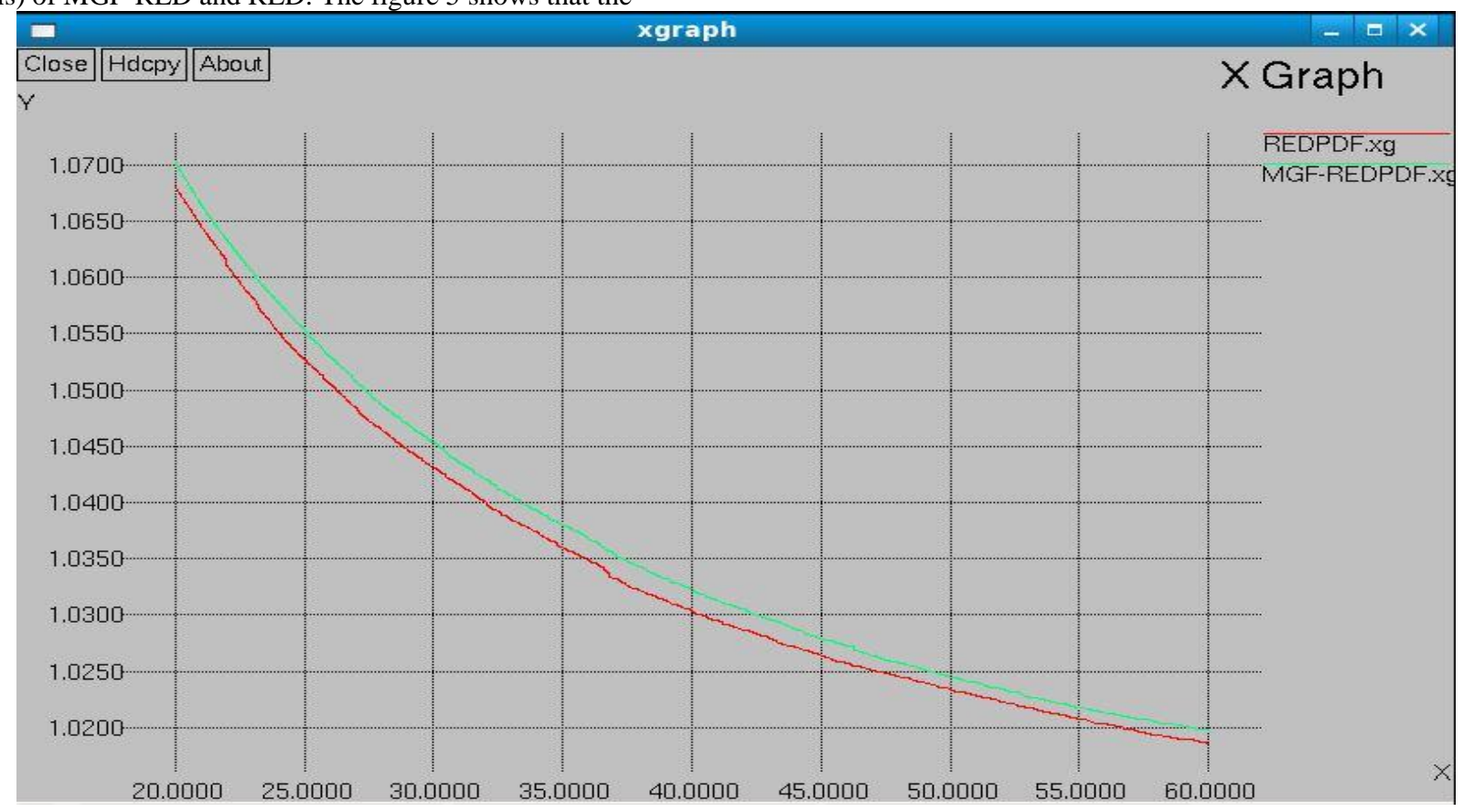

Fig 5: Packet Delivery Fraction versus simulation time

\section{Packet Dropped}

It may be defined as the total number of sent and forwarded packets minus the received packets. Figure 6 shows the simulation time (X-Axis) versus packet dropped (Y-Axis) of MGF-RED and RED algorithm. The figure 6 shows that the proposed algorithm gives the higher packet delivery ratio than the traditional RED. After $60 \mathrm{sec}$ of simulation, the packet delivery fraction is also increased from 1.0186 (RED) to 1.0197(MGF-RED).

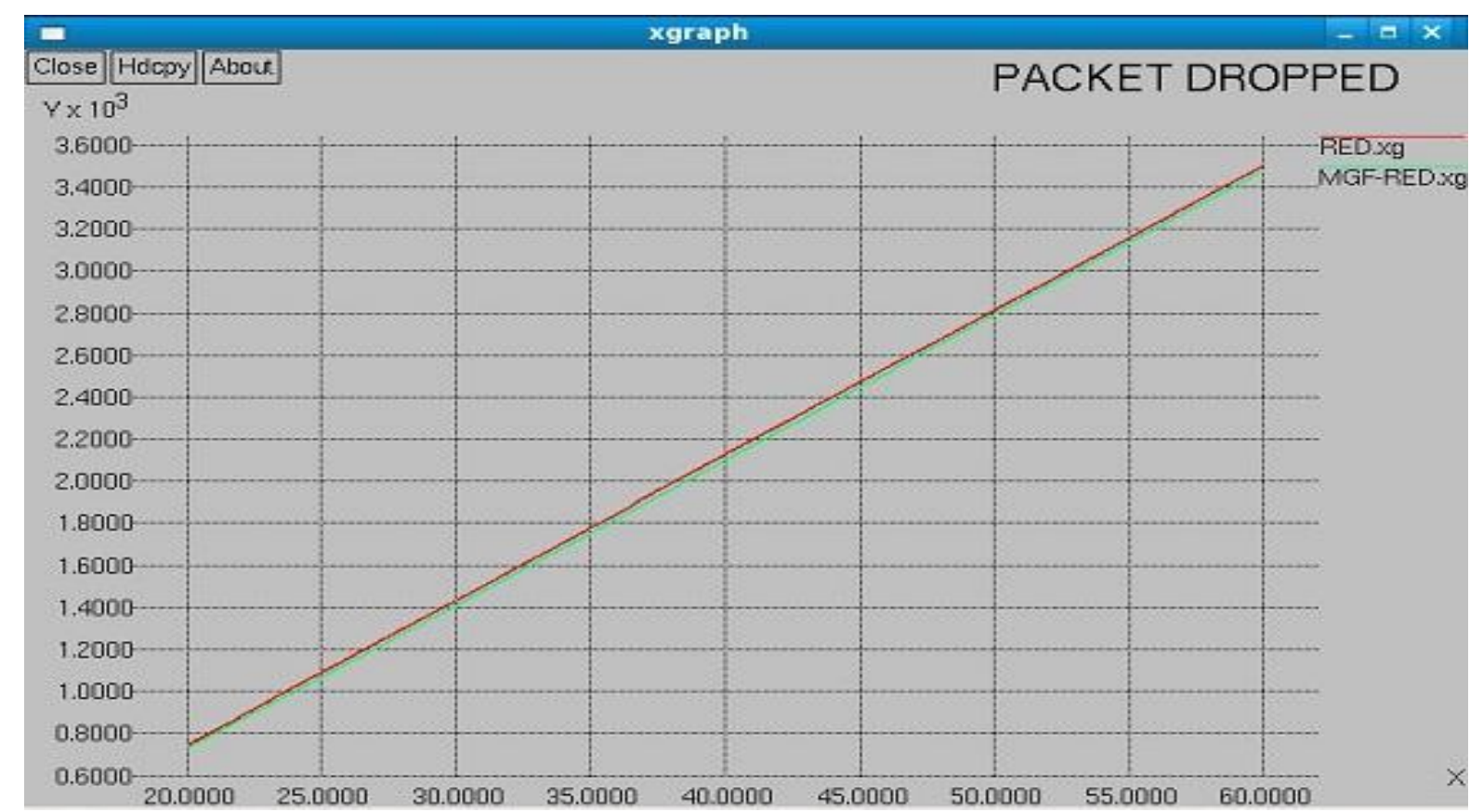

Fig 6: Number of Packet Dropped Versus Simulation Time 


\section{CONCLUSION}

This paper have proposed a new active queue management technique named as modified Gaussian function based random early detection algorithm (MGF-RED). The traditional RED calculates the packet drop probability based on linear function. In this algorithm the probability of packet drop increases as the average queue size increases. The propose work will generate a new probability function based on modified Gaussian probability function for calculating the packet drop probability. Simulations have been carried out and results shows that the proposed technique gives higher throughput, packet delivery fraction and lower number of dropped packets. In future the proposed work can be implemented for varying network load.

\section{REFERENCES}

[1] Geethu Mohandas, D. S. (2013). Survey on Routing Protocols on Mobile Adhoc. IEEE (pp. 514-517). IEEE.

[2] Bhabani Sankar Gouda, A. K. (2013). A Comprehensive Performance Analysis of Energy Efficient Routing Protocols in different traffic based Mobile Ad-hoc Networks. (pp. 306-312). IEEE

[3] HUAIZHI LI, M. S. (2007). ABRP: Anchor-based Routing Protocol for Mobile Ad Hoc Networks. Wireless Personal Communications (Springer) (C2006 , 277-300.

[4] Jeetendra Kumar Patel, J. D. (2012). Mobile Ad hoc Network Performance Improvement Using Strategical RED. IEEE.

[5] Rahul Verma, A. I. (2003). Active Queue Management using Adaptive RED. JOURNAL OF COMMUNICATIONS AND NETWORKS , 275-281.
[6] Sarita Simaiya, A. S. (2013). Performance Improvement of MANET by IRED. Journal of Environmental Science, Computer Science and Engineering \& Technology , 899907.

[7] Hussein Abdel-jaber, M. M. (2008). Fuzzy Logic Controller of Random Early Detection based on Average Queue Length and Packet Loss Rate. SPECTS, (pp. 428 432).

[8] Cao Zhen-zhen, X. Y. (2006). PERED:A New AQM Algorithm. ICWMMN.

[9] S. Athuraliya, S. Low, V. Li, and Q. Yin, REM: Active queue management, IEEE Network Magazine, vol. 15, pp. 48-53, May 2001.

[10] Floyd, Sally, Jacobson, Random Early Detection (RED) gateways for Congestion Avoidance. IEEE/ACM Transactions on Networking 1 (4): 397-413. doi:10.1109/90.251892. August 1993.

[11] Ganesh Patil,Sally McClean and Gaurav Raina. Drop tail and RED queue management with small buffers: Stability and hopf bifurcation. ICTACT journal on communication technology, june 2011.

[12] Guan-Yi Su, C. C.Random Early Detection Improved by Progressive Adjustment Method. Proceedings of IEEE 2008 6th National Conference on Telecommunication Technologies and IEEE 2008 2nd Malaysia Conference on Photonics, 26-27 August 2008, Putrajaya, Malaysia (pp. 250-253). IEEE. 